\title{
Effects of a chiral three-nucleon force on nucleus-nucleus scattering
}

\author{
Kosho Minomo, ${ }^{1, *}$ Masakazu Toyokawa, ${ }^{2}$ Michio Kohno, ${ }^{3,1}$ and Masanobu Yahiro ${ }^{2}$ \\ ${ }^{I}$ Research Center for Nuclear Physics, Osaka University, Ibaraki 567-0047, Japan \\ ${ }^{2}$ Department of Physics, Kyushu University, Fukuoka 812-8581, Japan \\ ${ }^{3}$ Physics Division, Kyushu Dental University, Kitakyushu 803-8580, Japan
}

(Dated: July 21, 2018)

\begin{abstract}
We investigate the effects of chiral NNLO three-nucleon force (3NF) on nucleus-nucleus elastic scattering, using a standard prescription based on the Brueckner-Hartree-Fock method and the $g$-matrix folding model. The $g$-matrix calculated in nuclear matter from the chiral $\mathrm{N}^{3} \mathrm{LO}$ two-nucleon forces $(2 \mathrm{NF})$ is close to that from the Bonn-B 2NF. Because the Melbourne group have already developed a practical $g$-matrix interaction by localizing the nonlocal $g$-matrix calculated from the Bonn-B 2NF, we consider the effects of chiral $3 \mathrm{NF}$, in this first attempt to study the $3 \mathrm{NF}$ effects, by modifying the local Melbourne $g$-matrix according to the difference between the $g$-matrices of the chiral $2 \mathrm{NF}$ and $2 \mathrm{NF}+3 \mathrm{NF}$. For nucleus-nucleus elastic scattering, the $3 \mathrm{NF}$ corrections make the folding potential less attractive and more absorptive. The latter novel effect is due to the enhanced tensor correlations in triplet channels. These changes reduce the differential cross section at the middle and large angles, improving the agreement with the experimental data for ${ }^{16} \mathrm{O}-{ }^{16} \mathrm{O}$ scattering at 70 $\mathrm{MeV} /$ nucleon and ${ }^{12} \mathrm{C}^{-12} \mathrm{C}$ scattering at $85 \mathrm{MeV} /$ nucleon.

PACS numbers: 21.30.Fe, 24.10.Ht, 25.70.Bc
\end{abstract}

Introduction. How important are three-nucleon forces (3NFs) in nuclear physics? This is one of the most important issues. This long-standing subject started with the $2 \pi$ exchange 3NF proposed by Fujita and Miyazawa [1]. For light nuclei, attractive 3 NFs were introduced to reproduce the binding energies [2]. In symmetric nuclear matter, meanwhile, repulsive 3 NFs were introduced to explain empirical saturation properties; for example see Refs. [3-6].

It is quite hard to determine $2 \mathrm{NF}$ and $3 \mathrm{NF}$ phenomenologically. This problem can be solved by chiral effective field theory (Ch-EFT) [7-10], since the theory systematically organizes interactions among nucleons. The roles of chiral 3NF was recently analyzed in light nuclei [11-14]. For symmetric nuclear matter, the $g$-matrix calculated from chiral $\mathrm{N}^{3} \mathrm{LO} 2 \mathrm{NF}$ plus NNLO 3NF [7, 9] well explain the empirical saturation properties with no adjustable parameter [15-18]. Scattering and reaction phenomena are important processes to explore $3 \mathrm{NF}$ effects.

Microscopic understanding of nucleon-nucleus and nucleus-nucleus scattering is another important subject in nuclear physics. The standard method of describing the scattering is the $g$-matrix folding model [19-24] with the local-density approximation. In the framework, the $3 \mathrm{NF}$ effects appear through the density dependence of the $g$-matrix. The CEG07 $g$-matrix is constructed from the $2 \mathrm{NF}$ based on the extended soft core model $[25,26]$ and $3 \mathrm{NF}$ effects are empirically taken into account by introducing the densitydependent vector-meson mass that reproduces empirical saturation properties of symmetric nuclear matter [27-29]. In the $g$-matrix calculation of Ref. [30], meanwhile, phenomenological 3NFs such as Urbana IX [31] and the three-nucleon interaction model [32, 33] are added to AV18 2NF [34]. Since these phenomenological $3 \mathrm{NFs}$ are also introduced to

*minomo@rcnp.osaka-u.ac.jp reproduce saturation properties of symmetric nuclear matter, they make the $g$-matrix less attractive and less absorptive at densities $(\rho)$ higher than the normal density $\rho_{0}$. The $3 \mathrm{NF}$ effects then reduce the folding potential $U(R)$ particularly at small distances $(R)$. For nucleus-nucleus elastic scattering, the $3 \mathrm{NF}$ effects are remarkable particularly at the middle and large angles where projectile and target densities are largely overlapped, although the strength of the imaginary part of $U(R)$ is adjusted to reproduce the experimental data in the analyses [29]. The question to be addressed now is how the effects of chiral $3 \mathrm{NF}$ appear in nucleus-nucleus elastic scattering.

In this Rapid Communication, we investigate the effects of chiral NNLO $3 \mathrm{NF}$ on nucleus-nucleus scattering, using the Brueckner-Hartree-Fock (BHF) method for nuclear matter and the $g$-matrix folding model for the scattering. In the nuclear matter calculations, furthermore, 3NFs are treated with the mean field approximation [32, 35-37], i.e., by averaging $3 \mathrm{NFs}$ over the third nucleon in the Fermi sea, since it is quite hard to treat three-body correlations in nuclear matter. This approximation is considered to be good for the low transferred-momentum components of the $g$-matrix. We then present the following simple prescription, as the first estimate of chiral-3NF effects on nucleus-nucleus scattering.

For nuclear matter, the single-particle potential $\mathcal{U}$ is determined from the diagonal component of the $g$-matrix. The $\mathcal{U}$ calculated from chiral $2 \mathrm{NF}$ is close to that from Bonn-B $2 \mathrm{NF}$ [38]. Hence, the effects of chiral $3 \mathrm{NF}$ can be expressed with the ratio of the $\mathcal{U}$ calculated from chiral $2 \mathrm{NF}+3 \mathrm{NF}$ to that from Bonn-B 2NF. For finite nuclei, the Melbourne group already presents a practical $g$-matrix interaction by localizing the nonlocal $g$-matrix calculated from Bonn-B 2NF [23]. The Melbourne $g$-matrix is highly reliable for nucleon-nucleus scattering where the $3 \mathrm{NF}$ effects are considered to be small [30], since the folding potential with the Melbourne $g$-matrix well reproduces the experimental data with no adjustable parameter $[23,39,40]$. The effects of chiral $3 \mathrm{NF}$ 
are then introduced to the local Melbourne $g$-matrix by multiplying it by the ratio. With the simple prescription, we make a qualitative discussion about the effects of chiral $3 \mathrm{NF}$ on nucleus-nucleus scattering. We consider ${ }^{16} \mathrm{O}-{ }^{16} \mathrm{O}$ scattering at $70 \mathrm{MeV} /$ nucleon and ${ }^{12} \mathrm{C}-{ }^{12} \mathrm{C}$ scattering at $85 \mathrm{MeV} /$ nucleon, since chiral EFT is good at lower incident energies $(E)$.

We first investigate the effects of chiral NNLO 3NF on symmetric nuclear matter through the $\mathcal{U}$ that corresponds to the folding potential $U(R)$ in nucleus-nucleus elastic scattering, and calculate $U(R)$ from the Melbourne interaction with chiral-3NF corrections to analyze the effects on nucleusnucleus scattering.

BHF calculations. Following Ref. [18], we evaluate the single-particle potential with the BHF method for positive energy $E$ from chiral $\mathrm{N}^{3} \mathrm{LO} 2 \mathrm{NF}$ plus NNLO $3 \mathrm{NF}$ with the cutoff energy $\Lambda=550 \mathrm{MeV}[7,9]$. Here the chiral NNLO 3NF $V_{123}$ is reduced to an effective $2 \mathrm{NF} V_{12(3)}$ with the meanfield approximation, that is, by integrating the $3 \mathrm{NF}$ over the third nucleon, and nuclear matter calculations are done from the combination of $\mathrm{N}^{3} \mathrm{LO} 2 \mathrm{NF} V_{12}$ and $V_{12(3)}$ in the standard manner.

The single-particle potential $\mathcal{U}^{S T}\left(k_{\mathrm{F}}, E\right)$ thus obtained depends on $E$, total spin $(S)$ and total isospin $(T)$ of two nucleons and the Fermi momentum $\hbar k_{\mathrm{F}}$. The potential is related to the $g$-matrix $G^{S T}$ as

$$
\begin{aligned}
\mathcal{U}^{S T}\left(k_{\mathrm{F}}, E\right)= & \sum_{\boldsymbol{k}^{\prime}}^{k_{\mathrm{F}}}\left\langle\boldsymbol{k} \boldsymbol{k}^{\prime}\right| G^{S T} \\
& +\frac{1}{6} V_{12(3)}^{S T}\left(1+\frac{Q}{E-H} G^{S T}\right)\left|\boldsymbol{k} \boldsymbol{k}^{\prime}\right\rangle_{A}
\end{aligned}
$$

where $\hbar \boldsymbol{k}$ and $\hbar \boldsymbol{k}^{\prime}$ are momenta of correlated two nucleons, the subscript |\rangle$_{\mathcal{A}}$ means the antisymmetrization between the correlated nucleons, and $Q /(E-H)$ is the nucleon propagator including the Pauli exclusion operator $Q$; see Ref. [18] for detail. The momentum $\hbar k$ is related to $E$ as $E=(\hbar \boldsymbol{k})^{2} /(2 m)+\operatorname{Re} \mathcal{U}$ for the nucleon mass $m$. The potential $\mathcal{U}^{S T}$ is mainly determined from the first term $G^{S T}$ on the right hand side of Eq. (1). For example at the normal density, the contribution of the first term is ten times as large as that of the second term having $V_{12(3)}$. In the first term, only the central part of the on-shell component of $G^{S T}$ contributes to the $\mathcal{U}^{S T}$, but the tensor part of $V_{12}$ and $V_{12(3)}$ affects the central part significantly. The mean-field approximation taken is good for the $\mathcal{U}^{S T}$ that is mainly determined from the on-shell component of $G^{S T}$.

Results of BHF calculations. Figure 1 displays $k_{\mathrm{F}}$ dependence of $\mathcal{U}^{S T}\left(k_{\mathrm{F}}, E\right)$ at $E=70 \mathrm{MeV}$ for each $S$ - $T$ channel. The results of chiral 2NF (squares) are close to those of Bonn$\mathrm{B} 2 \mathrm{NF}$ (triangles) for all the channels except the ${ }^{3} \mathrm{O}$ channel. For the real part of $\mathcal{U}^{S T}$ in the ${ }^{3} \mathrm{O}$ channel, the difference between the two results is not small particularly at high densities, but it little affects angular distributions of elastic cross sections, since the magnitude itself is small there.

Comparing the results of chiral $2 \mathrm{NF}+3 \mathrm{NF}$ (circles) with those of chiral 2NF (squares), one can see appreciable changes for the real part of $\mathcal{U}^{S T}$ in the ${ }^{1} \mathrm{E}$ channel and for the real and imaginary parts in the triplet channels $\left({ }^{3} \mathrm{E}\right.$ and $\left.{ }^{3} \mathrm{O}\right)$. Chiral

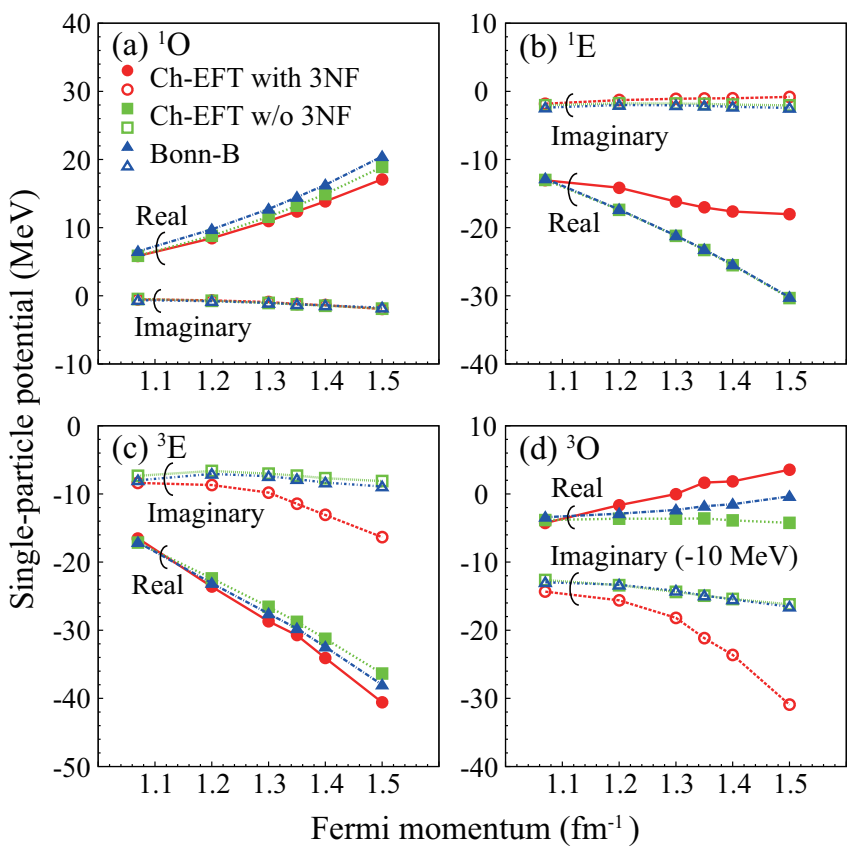

FIG. 1: (Color online) $k_{\mathrm{F}}$ dependence of the single-particle potentials at $E=70 \mathrm{MeV}$ for (a) ${ }^{1} \mathrm{O}$, (b) ${ }^{1} \mathrm{E}$, (c) ${ }^{3} \mathrm{E}$, and (d) ${ }^{3} \mathrm{O}$ channels. The circles, squares, and triangles stand for the results of chiral $2 \mathrm{NF}+3 \mathrm{NF}$, chiral $2 \mathrm{NF}$ and Bonn-B 2NF, respectively. The closed (open) symbols correspond to the real (imaginary) part of the singleparticle potentials. For ${ }^{3} \mathrm{O}$, the imaginary part is shifted down by 10 $\mathrm{MeV}$.

$3 \mathrm{NF}$ thus makes $\mathcal{U}^{S T}$ much less attractive for ${ }^{1} \mathrm{E}$. This effect has been known to be caused by the suppression of transitions to $\Delta$ resonance due to Pauli blocking. For the triplet channels, the $3 \mathrm{NF}$ enhances tensor correlations, and thereby makes $\mathcal{U}^{S T}$ much more attractive and absorptive for ${ }^{3} \mathrm{E}$ and more absorptive but less attractive for ${ }^{3} \mathrm{O}$. As the net effect of all the channels, the $\mathcal{U}^{S T}$ becomes much more absorptive because of tensor correlations enhanced by chiral 3NF, and becomes less attractive because of the suppression of transitions to $\Delta$ resonance in ${ }^{1} \mathrm{E}$. The enhancement of tensor correlations due to chiral $3 \mathrm{NF}$ is thus essential in addition to the repulsive nature of chiral 3NF itself. In the region $k_{\mathrm{F}} \lesssim 1.1 \mathrm{fm}^{-1}$ corresponding to $\rho \lesssim \rho_{0} / 2$, chiral 3NF yields little effect. Such 3NF effects on the $\mathcal{U}^{S T}$ directly reflect nucleus-nucleus scattering through the folding potential $U(R)$, as shown latter. This is natural, because $\mathcal{U}^{S T}$ in nuclear matter plays the same role as $U(R)$ in nucleus-nucleus scattering.

The Melbourne group constructed the local $g$-matrix interaction $g^{S T}\left(s ; k_{\mathrm{F}}, E\right)$ from Bonn-B 2NF and showed that the interaction is quite practical and reliable for nucleonnucleus scattering [23], where $s$ is the coordinate between correlated nucleons. We then introduce $3 \mathrm{NF}$ corrections to $g^{S T}\left(s ; k_{\mathrm{F}}, E\right)$ in the following manner, since the $\mathcal{U}^{S T}$ calculated from Bonn-B 2NF well simulate the results of chiral $\mathrm{N}^{3} \mathrm{LO} 2 \mathrm{NF}$ :

$$
g^{S T}\left(s ; k_{\mathrm{F}}, E\right) \rightarrow f^{S T}\left(k_{\mathrm{F}}, E\right) g^{S T}\left(s ; k_{\mathrm{F}}, E\right)
$$


with the factor $f^{S T}\left(k_{\mathrm{F}}, E\right)$ defined by

$$
f^{S T}\left(k_{\mathrm{F}}, E\right)=\mathcal{U}_{(2 \mathrm{NF}+3 \mathrm{NF})}^{S T}\left(k_{\mathrm{F}}, E\right) / \mathcal{U}_{(2 \mathrm{NF})}^{S T}\left(k_{\mathrm{F}}, E\right),
$$

where $\mathcal{U}_{(2 \mathrm{NF}+3 \mathrm{NF})}^{S T}\left(k_{\mathrm{F}}, E\right)$ and $\mathcal{U}_{(2 \mathrm{NF})}^{S T}\left(k_{\mathrm{F}}, E\right)$ stand for the single-particle potentials with and without $3 \mathrm{NF}$, respectively. This prescription mainly takes account of the effects of the on-shell component of chiral 3NF on the local Melbourne $g$ matrix interaction.

Folding-model calculations. Now we consider nucleusnucleus scattering with the folding model. In the model, the folding potential $U(R)$ is obtained by folding $g^{S T}\left(s ; k_{\mathrm{F}}, E\right)$ with projectile and target densities in the standard way [24, $29,41-43]$, where the Fermi momentum $\hbar k_{\mathrm{F}}$ is replaced by a local Fermi momentum that is evaluated with the frozen density approximation. That is, $k_{\mathrm{F}}$ is estimated from the sum of projectile and target local densities at the middle point of interacting nucleons. As the projectile and target proton densities, we take the phenomenological ones determined from the electron scattering [44], applying proton finite-size corrections to the densities with the standard manner [45]. The neutron densities are assumed to be the same as the proton ones, which is accurate enough for light nuclei.

Results of folding-model calculations. Figure 2 shows the folding potential $U(R)$ for ${ }^{16} \mathrm{O}-{ }^{16} \mathrm{O}$ elastic scattering at 70 $\mathrm{MeV} /$ nucleon. The solid (dashed) curve represents the result with (without) $3 \mathrm{NF}$ corrections. The potential is mainly determined from the even-channel $\left({ }^{1} \mathrm{E}\right.$ and $\left.{ }^{3} \mathrm{E}\right)$ components, since the odd-channel $\left({ }^{1} \mathrm{O}\right.$ and $\left.{ }^{3} \mathrm{O}\right)$ components are almost canceled after the sum of the direct and exchange parts of $U(R)$. For the real part of $U(R)$, chiral 3NF works as a repulsive force, but it is weak because of the offset between the repulsive contribution in ${ }^{1} \mathrm{E}$ and the attractive one in ${ }^{3} \mathrm{E}$. For the imaginary part, chiral 3NF makes $U(R)$ strongly absorptive, since the contribution works additively between ${ }^{3} \mathrm{E}$ and ${ }^{3} \mathrm{O}$ channels.

In the previous works of Refs. [27-30], the phenomenological 3NFs work repulsively and less absorptively. For the real part of $U(R)$, the present result is consistent with the previous ones qualitatively, although the repulsive effect is small in the present work but large in the previous works. For the imaginary part, meanwhile, chiral $3 \mathrm{NF}$ enhances the absorption of $U(R)$, in contrast to the opposite effect of phenomenological $3 \mathrm{NFs}$. The difference comes from the fact that chiral $3 \mathrm{NF}$ enhances tensor correlations but the phenomenological 3NFs do not.

Figure 3 shows differential cross sections for (a) ${ }^{16} \mathrm{O}-{ }^{16} \mathrm{O}$ elastic scattering at $70 \mathrm{MeV} /$ nucleon and (b) ${ }^{12} \mathrm{C}-{ }^{12} \mathrm{C}$ elastic scattering at $85 \mathrm{MeV} /$ nucleon as a function of scattering angle $\theta$ in the center of mass system. The solid (dashed) curve corresponds to the result of Melbourne interaction with (without) chiral-3NF corrections. Chiral 3NF reduces the cross sections in middle and large angles $\left(\theta>5^{\circ}\right)$ and hence improves the agreement with the experimental data [46, 47]. Switching off chiral-3NF effects for either the real or the imaginary part of $U(R)$, we find that the interference between the two effects is important at the middle and large angles.

For the ${ }^{16} \mathrm{O}-{ }^{16} \mathrm{O}$ scattering, the result of chiral $2 \mathrm{NF}+3 \mathrm{NF}$ slightly overestimates the experimental data. If the real part
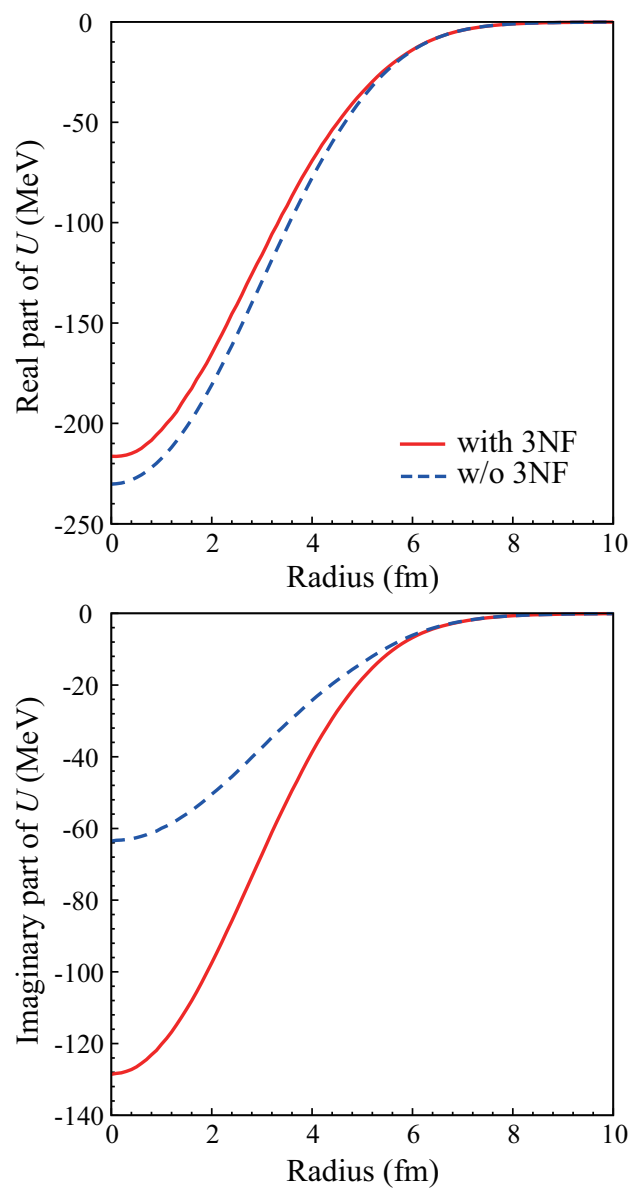

FIG. 2: (Color online) The folding potential for ${ }^{16} \mathrm{O}-{ }^{16} \mathrm{O}$ elastic scattering at $70 \mathrm{MeV} /$ nucleon. The real and imaginary parts of the potential are shown in the upper and lower panels, respectively. The solid (dashed) curve represents the result with (without) $3 \mathrm{NF}$ effects.

of the potential is reduced by $10 \%$ and the imaginary part is enhanced by $10 \%$, the result can reproduce the data. Since the correction is rather small, as an origin of the difference we can consider higher-order corrections such as off-shell corrections due to chiral $3 \mathrm{NF}$ and corrections for the mean-field approximation. For the ${ }^{12} \mathrm{C}-{ }^{12} \mathrm{C}$ scattering, the diffraction pattern is slightly shifted forward in the result of chiral $2 \mathrm{NF}+3 \mathrm{NF}$ compared with the data. The difference may come from projectile and/or target excitations, since the previous work of Ref. [48] shows that the excitation effects slightly shift the diffraction pattern backward. The higher-order corrections mentioned above may be masked by the excitation effects. Further analysis along this line is quite interesting as a future work.

Total reaction cross sections $\sigma_{\mathrm{R}}$ are mainly determined from the imaginary part of $U(R)$. We then check whether the present potential with $3 \mathrm{NF}$ corrections reproduces measured $\sigma_{\mathrm{R}}$. Table I shows $\sigma_{\mathrm{R}}$ for ${ }^{16} \mathrm{O}-{ }^{16} \mathrm{O}$ scattering at 70 $\mathrm{MeV} /$ nucleon and ${ }^{12} \mathrm{C}^{12}{ }^{12} \mathrm{C}$ scattering at $85 \mathrm{MeV} /$ nucleon. The experimental data are available for the ${ }^{12} \mathrm{C}-{ }^{12} \mathrm{C}$ scattering, but not for the ${ }^{16} \mathrm{O}_{-}{ }^{16} \mathrm{O}$ scattering. Chiral $3 \mathrm{NF}$ enhances $\sigma_{\mathrm{R}}$ only by a few percent, since the effects appear 

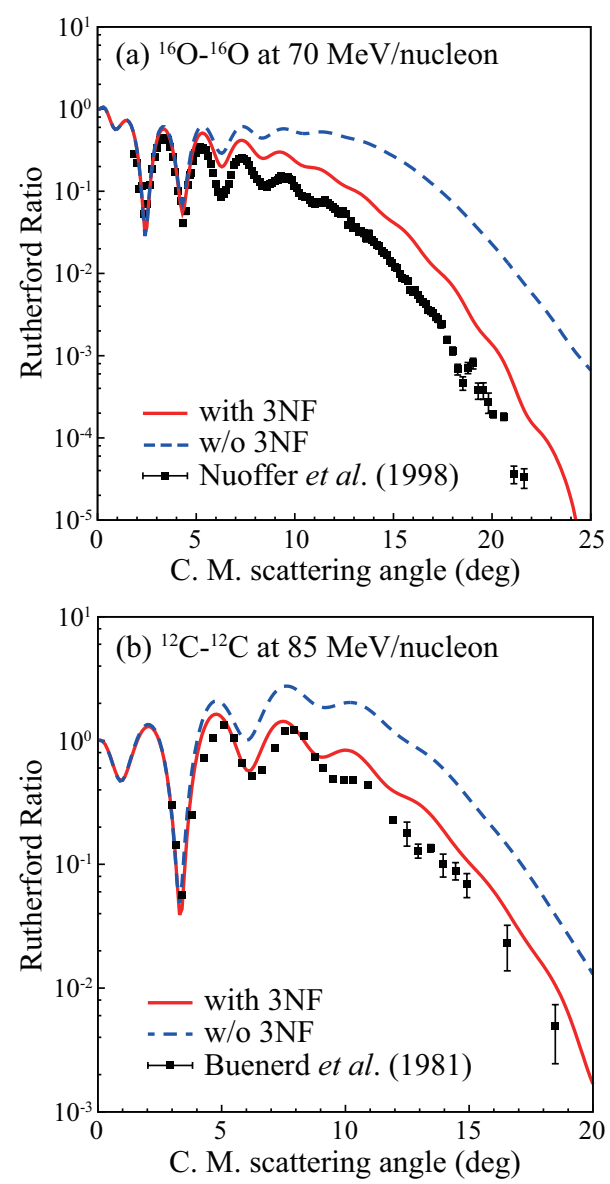

FIG. 3: (Color online) The differential cross sections of (a) ${ }^{16} \mathrm{O}$ ${ }^{16} \mathrm{O}$ elastic scattering at $70 \mathrm{MeV} /$ nucleon and (b) ${ }^{12} \mathrm{C}-{ }^{12} \mathrm{C}$ elastic scattering at $85 \mathrm{MeV} /$ nucleon as a function of the scattering angle in the center of mass system. The solid (dashed) line corresponds to the result of Melbourne interaction with (without) $3 \mathrm{NF}$ corrections. The experimental data are taken from Refs. [46, 47].

only at small $R$ where $U(R)$ already has strong absorption. The calculated result slightly overestimates the experimental data [49], but it is not serious at all. The Melbourne $g$-matrix interaction with $3 \mathrm{NF}$ corrections is thus reliable also for $\sigma_{\mathrm{R}}$.

TABLE I: Total reaction cross sections for nucleus-nucleus scattering around $70 \mathrm{MeV} /$ nucleon (in the unit of $\mathrm{mb}$ ). The experimental data is taken from Ref. [49].

\begin{tabular}{cccc}
\hline \hline system & with 3NF & w/o 3NF & Exp. [49] \\
\hline${ }^{16} \mathrm{O}-{ }^{16} \mathrm{O} @ 70 \mathrm{MeV} /$ nucleon & 1423 & 1401 & - \\
${ }^{12} \mathrm{C}-{ }^{12} \mathrm{C} @ 85 \mathrm{MeV} /$ nucleon & 1025 & 989 & $998 \pm 13$ \\
\hline \hline
\end{tabular}

Summary. We have made a qualitative discussion about the effects of chiral NNLO 3 NF on nucleus-nucleus elastic scattering, using the simple prescription of Eqs. (2) and (3). In this prescription, on-shell corrections due to chiral $3 \mathrm{NF}$ are introduced to the local Melbourne $g$-matrix interaction. It is to be stressed that we have not introduced any ad hoc phenomenological adjustment.

For nuclear matter, chiral 3NF makes the single-particle potentials less attractive for the ${ }^{1} \mathrm{E}$ channel and more absorptive for the triplet channels. The effects mainly comes from the $2 \pi$ exchange process. For the triplet channels, the diagram enhances tensor correlations and hence the absorption. These $3 \mathrm{NF}$ corrections are incorporated in the folding potential for nucleus-nucleus scattering by modifying the local Melbourne $g$-matrix interaction by the multiplicative factor prescribed as Eqs. (2) and (3). The corrections make the folding potential less attractive and more absorptive. Since the folding potential is mainly determined from the ${ }^{1} \mathrm{E}$ - and ${ }^{3} \mathrm{E}$ channel components, we can conclude that the repulsive correction to the folding potential comes from the suppression of transitions to $\Delta$ resonance and the absorptive correction is originated in the enhancement of tensor correlations. The two effects reduce differential elastic cross sections for both ${ }^{16} \mathrm{O}$ ${ }^{16} \mathrm{O}$ scattering at $70 \mathrm{MeV} /$ nucleon and ${ }^{12} \mathrm{C}-{ }^{12} \mathrm{C}$ scattering at $85 \mathrm{MeV} /$ nucleon. Eventually, chiral 3NF improves the agreement with the experimental data.

It is instructive to note the qualitative difference of the present result from other similar studies in the literature. In the previous works of Refs. [27-30], the phenomenological 3 NFs make the folding potential $U(R)$ less attractive and less absorptive. The present result is consistent with the previous ones qualitatively for the real part of $U(R)$, although the repulsive effect is small in the present work but large in the previous works. For the imaginary part, meanwhile, chiral 3NF makes $U(R)$ more absorptive, whereas the phenomenological $3 \mathrm{NFs}$ do $U(R)$ less absorptive. The difference comes from the fact that chiral $3 \mathrm{NF}$ enhances tensor correlations but the phenomenological 3NFs do not.

The on-shell corrections due to chiral 3NF surely improve the agreement with the experimental data on nucleus-nucleus elastic scattering, but the agreement is not perfect. This may come from higher-order corrections such as off-shell corrections due to chiral $3 \mathrm{NF}$ and corrections for the mean-field approximation. Further analysis along this line toward deeper microscopic understanding of nucleus-nucleus scattering is quite interesting as a future work.

\section{Acknowledgements}

This work is supported in part by by Grant-in-Aid for Scientific Research (Nos. 244137, 25400266, and 26400278) from Japan Society for the Promotion of Science (JSPS). 
[1] J. Fujita and H. Miyazawa, Prog. Theor. Phys. 17, 366 (1957).

[2] R. B. Wiringa and S. C. Pieper, Phys. Rev. Lett. 89, 182501 (2002).

[3] R. B. Wiringa, V. Fiks, and A. Fabrocini, Phys. Rev. C 38, 1010 (1988).

[4] H. Müther and A. Polls, Prog. Part. Nucl. Phys. 45, 243 (2000).

[5] Y. Dewulf, W. H. Dickhoff, D. Van Neck, E. R. Stoddard, and M. Waroquier, Phys. Rev. Lett. 90, 152501 (2003).

[6] S. K. Bogner, A. Schwenk, R. J. Furnstahl, and A. Nogga, Nucl. Phys. A 763, 59 (2005).

[7] E. Epelbaum, W. Glöckle, and Ulf-G. Meißner, Nucl. Phys. A747, 362 (2005).

[8] E. Epelbaum, H.-W. Hammer, and Ulf-G. Meißner, Rev. Mod. Phys. 81, 1773 (2009).

[9] K. Hebeler, S. K. Bogner, R. J. Furnstahl, A. Nogga, and A. Schwenk, Phys. Rev. C 83, 031301(R) (2011).

[10] R. Machleidt and D. R. Entem, Phys. Rep. 503, 1 (2011).

[11] E. Epelbaum, A. Nogga, W. Glöckle, H. Kamada, Ulf-G. Meißner, and H. Witała, Phys. Rev. C 66, 064001 (2002).

[12] A. Nogga, P. Navrátil, B. R. Barrett, and J. P. Vary, Phys. Rev. C 73, 064002 (2006).

[13] P. Navrátil, V. G. Gueorguiev, J. P. Vary, W. E. Ormand, and A. Nogga, Phys. Rev. Lett. 99, 042501 (2007).

[14] R. Skibiński et al., Phys. Rev. C 84, 054005 (2011).

[15] F. Sammarruca, B. Chen, L. Coraggio, N. Itaco, and R. Machleidt, Phys. Rev. C 86, 054317 (2012).

[16] M. Kohno, Phys. Rev. C 86, 061301(R) (2012).

[17] A. Carbone, A. Polls, and A. Rios, Phys. Rev. C 88, 044302 (2013).

[18] M. Kohno, Phys. Rev. C 88, 064005 (2013).

[19] J. P. Jeukenne, A. Lejeune and C. Mahaux, Phys. Rev. C16, 80 (1977).

[20] G. Bertsch, J. Borysowicz, H. McManus, and W.G. Love, Nucl. Phys. A284, 399(1977).

[21] F.A. Brieva and J.R. Rook, Nucl. Phys. A291, 299 (1977); ibid. 291, 317 (1977); ibid. 297, 206 (1978).

[22] N. Yamaguchi, S. Nagata and T. Matsuda, Prog. Theor. Phys. 70, 459 (1983); N. Yamaguchi, S. Nagata and J. Michiyama, Prog. Theor. Phys. 76, 1289 (1986).

[23] K. Amos, P. J. Dortmans, H. V. von Geramb, S. Karataglidis, and J. Raynal, Adv. Nucl. Phys. 25, 275 (2000).

[24] D. T. Khoa and G. R. Satchler, Nucl. Phys. A 668, 3 (2000).

[25] Th. A. Rijken, Phys. Rev. C 73, 044007 (2006).
[26] Th. A. Rijken and Y. Yamamoto, Phys. Rev. C 73, 044008 (2006).

[27] T. Furumoto, Y. Sakuragi, and Y. Yamamoto, Phys. Rev. C 78, 044610 (2008).

[28] T. Furumoto, Y. Sakuragi, and Y. Yamamoto, Phys. Rev. C 79, 011601(R) (2009).

[29] T. Furumoto, Y. Sakuragi, and Y. Yamamoto, Phys. Rev. C 80, 044614 (2009).

[30] S. Rafi, M. Sharma, D. Pachouri, W. Haider, and Y. K. Gambhir, Phys. Rev. C 87, 014003 (2013).

[31] B. S. Pudliner, V. R. Pandharipande, J. Carlson, S. C. Pieper, and R. B. Wiringa, Phys. Rev. C 56, 1720 (1997).

[32] B. Friedman and V. R. Pandharipande, Nucl. Phys. A 361, 502 (1981).

[33] I. E. Lagaris and V. R. Pandharipande, Nucl. Phys. A 359, 349 (1981).

[34] R. B. Wiringa, V. G. J. Stoks, and R. Schiavilla, Phys. Rev. C 51, 38 (1995).

[35] B. A. Loiseau, Y. Nogami, and C. K. Ross, Nucl. Phys. A 165, 601 (1971).

[36] T. Kasahara, Y. Akaishi, and H. Tanaka, Prog. Theor. Phys. Suppl. 56, 96 (1974).

[37] J. W. Holt, N. Kaiser, and W. Weise, Phys. Rev. C 81, 024002 (2010).

[38] R. Machleidt, K. Holinde, and Ch. Elster, Phys. Rep. 149, 1 (1987).

[39] K. Minomo, K. Ogata, M. Kohno, Y. R. Shimizu, and M. Yahiro, J. Phys. G 37, 085011 (2010)

[40] M. Toyokawa, K. Minomo, and M. Yahiro, Phys. Rev. C 88, 054602 (2013).

[41] B. Sinha, Phys. Rep. 20, 1 (1975).

[42] B. Sinha and S. A. Moszkowski, Phys. Lett. B 81, 289 (1979).

[43] G. R. Satchler and W. G. Love, Phys. Rep. 55, 183 (1979).

[44] H. de Vries, C. W. de Jager, and C. de Vries, At. Data Nucl. Data Tables 36, 495 (1987).

[45] R. P. Singhal, M. W. S. Macauley, and P. K. A. de Witt Huberts, Nucl. Instr. and Meth. 148, 113 (1978).

[46] F. Nuoffer et al., Nuovo Cimento A 111, 971 (1998).

[47] M. Buenerd et al., Phys. Lett. B 102, 242 (1981).

[48] T. Furumoto and Y. Sakuragi, Phys. Rev. C 87, 014618 (2013).

[49] M. Takechi et al., Phys. Rev. C 79, 061601(R) (2009). 